Chapman University

Chapman University Digital Commons

Pharmacy Faculty Articles and Research

School of Pharmacy

$12-2016$

\title{
Carriers for the Tunable Release of Therapeutics: Etymological Classification and Examples
}

Vuk Uskoković

Chapman University, uskokovi@chapman.edu

Shreya Ghosh

University of Illinois at Chicago

Follow this and additional works at: http://digitalcommons.chapman.edu/pharmacy_articles

Part of the Pharmaceutics and Drug Design Commons

\section{Recommended Citation}

Uskoković V, Ghosh S. Carriers for the tunable release of therapeutics: etymological classification and examples. Expert Opin Drug Deliv. 2016;13(12):1-13. doi:10.1080/17425247.2016.1200558

This Article is brought to you for free and open access by the School of Pharmacy at Chapman University Digital Commons. It has been accepted for inclusion in Pharmacy Faculty Articles and Research by an authorized administrator of Chapman University Digital Commons. For more information, please contact laughtin@chapman.edu. 


\section{Carriers for the Tunable Release of Therapeutics: Etymological Classification and Examples}

\section{Comments}

This is a pre-copy-editing, author-produced PDF of an article accepted for publication in Expert Opinion on Drug Delivery, volume 13, issue 12, in 2016. The definitive publisher-authenticated version is available online at DOI: $10.1080 / 17425247.2016 .1200558$

\section{Copyright}

Taylor \& Francis 


\title{
Carriers for the Tunable Release of Therapeutics: Etymological Classification and Examples
}

\author{
Vuk Uskoković ${ }^{1,2}$ and Shreya Ghosh ${ }^{1}$ \\ ${ }^{1}$ Department of Bioengineering, University of Illinois, Chicago, IL 60607-7052, USA \\ ${ }^{2}$ Department of Biomedical and Pharmaceutical Sciences, Chapman University, Irvine, CA \\ 92618-1908, USA
}

\begin{abstract}
Introduction-Physiological processes at the molecular level take place at precise spatiotemporal scales, which vary from tissue to tissue and from one patient to another, implying the need for the carriers that enable tunable release of therapeutics.

Areas Covered-Classification of all drug release to intrinsic and extrinsic is proposed, followed by the etymological clarification of the term "tunable" and its distinction from the term "tailorable". Tunability is defined as analogous to tuning a guitar string or a radio receiver to the right frequency using a single knob. It implies changing a structural parameter along a continuous quantitative scale and correlating it numerically with the release kinetics. Examples of tunable, tailorable and environmentally responsive carriers are given, along with the parameters used to achieve these levels of control.

Expert Opinion-Interdependence of multiple variables defining the carrier microstructure obstructs the attempts to elucidate parameters that allow for the independent tuning of release kinetics. Learning from the tunability of nanostructured materials and superstructured metamaterials can be a fruitful source of inspiration in the quest for the new generation of tunable release carriers. The greater intersection of traditional materials sciences and pharmacokinetic perspectives could foster the development of more sophisticated mechanisms for tunable release.
\end{abstract}

\section{Keywords}

Drug Delivery; Release Kinetics; Tailorable Release; Tunable Release

\section{Introduction}

The increasing incidence of chronic diseases coupled with the incentives to improve the quality of life has attributed to the development of advanced biomaterials[1][2][3]. Over the past several decades, metals, polymers, ceramics and bio-derived materials have been extensively studied and used as medical implants. Such materials can be designed for a wide

Corresponding author: Vuk Uskoković, vuk21@yahoo.com.

Contributions

V. Uskoković conceived the review study, wrote it and designed all figures and tables. S. Ghosh collected most examples for Table 2, a few examples for Table 3, and provided assistance with editing figures, tables and references. 
range of biomedical applications, e.g., controlled drug delivery devices, tissue engineering constructs, vascular grafts and multiple other therapeutic, regenerative and/or diagnostic platforms. However, the ability of materials scientists to fine-tune the structure of materials to desired mechanical or electromagnetic properties has surpassed the ability to fine-tune them to exhibit complex kinetics of release of therapeutic molecules.

Overall, there is a need for the development of ever more sensitive and smarter controlled release carriers in pharmaceutics. To terminate the drug release when the therapeutic goal has been achieved and to trigger the release from the carrier only in the presence of a certain disease marker are only some of the demands for the new generation of drug release carriers. At a finer level, the drug release kinetic profiles could be designed for precise temporal matching of the targeted physiological processes that facilitate healing on the molecular scale. This would prevent systemic or local toxicity, ensuring patient safety while also increasing the efficacy with which the device delivers its therapeutic payload.

Many biological processes take place at precise timescales. For example, the reaction of the complement system to a foreign entity involves a cascade of processes, each of which is preceded and anteceded by others at precise timescales. Sophisticated targeting of individual processes in such a cascade necessitates the initiation of the therapeutic action through the controlled release of the right molecular agent within a very specific time window. Another example comes from wound healing (Fig. 1a), a physical response to injury that similarly involves a multitude of processes, each of which occur within precise time windows. Interfering with these processes with the intended therapeutic effect means that the timescale of this interference must be precisely set to the right time windows. Bone remodeling is yet another biological process divisible to individual stages (Fig. 1b), implying that an ideal therapeutic interference with this process would also require a sequential, precisely temporally tuned delivery of the right therapeutic agents. Targeted drug delivery in terms of precise 3D location of the released drug would be greatly enriched if one such targeting effect in the fourth, temporal dimension is achieved too.

Types of compounds traditionally delivered with the use of sustained release platforms since the early days of controlled drug delivery devices are listed in Table 1. However, preclinical optimizations of ideal release kinetics to individual pathological features on the molecular scale have ceded place to "one size fits all" approach, where a single type of implant, microstructurally speaking, is being supplied to the clinic, ignoring the fact that every clinical case is unique and different from any other. Additionally, utilizing the therapeutic role of small biomolecules and proteins on tissue regeneration may demand their controlled release at precisely tunable timescales. Signaling molecules, including hormones, cytokines and growth factors are included on this long list of compounds whose finely tuned delivery from the kinetic standpoint would greatly facilitate the wound healing and tissue regeneration processes.

The need for tunable release profiles is illustratable by the dichotomy between cortical and cancellous bone. Namely, with the cortical bone being more compact and having a lower remodeling rate than the more porous and vascularized cancellous bone, the ideal release profiles should be faster and more intense in the treatment of cancellous bone. As one shifts 
from the surface of long bones to their marrow, one simultaneously moves in the direction of decreasing compactness and an increasing soft tissue nature. The clearance rate of the released drug would consequently decrease with the distance from the central axis of long bones; the need for the tuning of the release rate to the right implant location naturally follows from acknowledging this functionally gradient nature of bone. Sustained delivery of pain medication from sutures and wound dressings has, likewise, demonstrated that no single release profile fits all patients. Rather, in some cases the medication has to be supplemented orally because of the insufficient dose delivered, whereas in other cases the oral administration of opioid antagonists is necessary because of the adverse effects of the systemic overdose[19]. These clinical circumstances imply that tunable release agents are necessary to match the wide variety of analgesic needs of patients.

Therefore, the variability of structure and properties of a single tissue depending on the location as well as the variability of structure and properties of identical tissues in different patients imply the demand for carriers whose release rates could be tuned by varying a single parameter that defines the corresponding internal structure of the material across a continuous range of values. In such a manner, carriers would be designed to deliver drugs to tissues in a tunable fashion and accommodate directly to the ideal projected drug delivery profiles for the given tissues and the patient. In theory, rapid prototyping of implants in direct feedback with computerized axial tomography scans could thus be complemented with setting of the properties of the implant material to achieve the exactly desired release profile without resorting to qualitative modifications of the material. This qualitative constancy, notwithstanding the kinetic versatility, would also minimize the number of regulatory hurdles standing on the translational path. The applicative benefits of devising tunable drug carriers are thus clearly numerous enough to justify the furthering of the research on them.

\subsection{Elementary classification: intrinsic vs. extrinsic control of the release}

Kinetically controlled and tunable release of therapeutics from a carrier is attainable by multiple means. Still, all of them could be roughly divided to two categories: intrinsic and extrinsic. As shown in Fig. 2, in case of the intrinsic controlled release, the structure of the carrier becomes modified to vary the release rates and amounts, whereas in case of the extrinsic controlled release, either the environmental factors or the external, manual or automatized control triggers the release and occasionally enables the tuning of its rate to the desired kinetics. In what follows we will briefly mention the examples that belong to both, albeit for the most part limiting the scope of this review to the intrinsically tunable carriers.

\subsection{Etymological clarification: tailorable vs. tunable}

Confusion exists in the literature as to what the term "tunable" exactly means. Most occurrences of this attribute are incorrect, given that they describe the mere ability of a carrier to produce two or more distinct release profiles following the tweaking of its structure or introducing a variation in the physicochemical environment surrounding it. However, tunability, in the truer sense of the word, should be analogous to tuning a radio receiver along a continuous range of frequencies to a desired radio station or tuning a guitar string to the desired pitch. In other words, it should imply the correspondence between a 
continuous range of values of a specific structural parameter of the carrier and a similarly continuous range of values of properties determining the release of the drug from the given carrier.

For the sake of convention, we will ascribe the attribute "tailorable" to the process of controlling the release kinetics by means of introducing qualitative changes in the carrier. We will ascribe the attribute "tunable" to the process of controlling the release kinetics by varying the quantitative value of a parameter that defines the structure of the carrier on a continuous scale. For example, if the size of particles loaded with a drug is reduced and such a reduction entails an increased rate of release of the physisorbed drug as the result of increased surface-to-volume ratio, one such effect could receive the attribute of "tunable", provided that the control over the particle size is possible within a more or less continuous range and that a numerical correlation could be established between the particle size and the release rate. Likewise, when the stirring rate is varied to tune the nanoparticle size of coordination-metal polymeric particles within a wide range of values[20], such an effect, albeit somewhat trivial, is tunable so long as this range is continuous and broad enough. Changing the release profile by changing the chemical identity of the ligand, on the other hand, does not constitute a tunable effect, even though it is frequently reported in the literature as such. Since tailoring denotes the act of cutting and pasting different patches of clothes, controlling the release by the presence or absence of certain particle components can be seen as analogous to the act of tailoring.

Fig. 2 schematically depicts this difference between tailoring and tuning. In the case of tunable release, a particular compositional or structural parameter is tuned to give a desired release profile, whereas in the case of tailorable release, adding or subtracting different carrier components, analogous to copying and pasting different patches of clothes, is used to modify the release profile. For example, the yielding of distinct release profiles upon the qualitative modification of the phase composition of calcium phosphate nanoparticles as carriers could be considered tailorable (Fig. 3a) [21]. In contrast, the tuning of the release of vancomycin and ciprofloxacin to anywhere between zero hours and two weeks by changing the weight ratio between two components of a hydroxyapatite cement, differing only in the rate of transformation into apatite from the amorphous primary precipitate, presents an authentic instance of the use of calcium phosphate nanoparticles as tunable release carriers (Fig. 5a-b) [22].

Another class of carriers which are often inappropriately named "tunable" are those whose release kinetics takes different forms depending on a specific physicochemical parameter that defines the conditions of their environment. Unlike the authentically tunable release carriers, in which tunability originates from the intrinsic control over a specific structural, compositional or synthesis parameter, in the case of such "smart" carriers, which release their payload only upon receiving a specific environmental stimuli in the form of a disease marker, the release kinetics is controlled extrinsically, as it is being caused by the indiscrete variation of an environmental parameter. One could include in this category carriers whose release is controlled by external effects, such as heat, light, magnetic field, ultrasound, mechanical force, and electric current (Fig. 2). 


\subsection{Examples of tailorable carriers}

A study in which various material/drug combinations for wound healing were tested came to conclusion that incorporation of diclofenac into four fibrous wound dressing materials, namely alginate, sodium carboxymethyl cellulose ( $\mathrm{Na}-\mathrm{CMC})$, viscose rayon and poly(ethylene terephthalate) (PET) renders kinetically distinct release profiles that could be tailored for both chronic and acute wound treatments [23]. While alginate and Na-CMC exhibited prolonged release and were found to be applicable for the treatment of chronic wounds, viscose rayon and PET were suitable for acute wounds. Next, Fig. $3 \mathrm{~b}$ illustrates a difference in the release of vancomycin depending on the qualitative composition of cements composed of poly(D, L-lactide-co-glycolide) (PLGA) and calcium phosphate nanoparticles. While sole calcium phosphate caused the drug to be rapidly released, the addition of mannitol or PLGA, be it as empty or loaded microspheres, extended it to various degrees. Then, the release of fusion protein SH3-IGF-1 can be tailored by binding different peptides to a hydrogel composed of hyaluronan and methylcellulose (HAMC) as the carrier. As can be seen in Fig. 3c, the protein release is reduced in the presence of a strong binding peptide (SBP) as compared to unbound HAMC and HAMC with a weak binding peptide (WBP). Another example of tailorable release comes from the threefold acceleration of the release of diltiazem hydrochloride from chitosan nanoparticles following the conjugation of chitosan with L-leucine[24]. Adjusting the release of doxorubicin or vincristine to a desired rate by modifying silk films as the carrier with carboxyl or sulfonate groups also belongs to the category of tailored release (Fig. 3d).

\subsection{Examples of devices with environmentally sensitive, stimuli-responsive release}

\subsubsection{Carriers stimulated by the chemical conditions of their}

microenvironment-Carriers capable of releasing their therapeutic payload only in the presence of a particular disease marker present the first step toward the development of theranostic drug delivery platforms. One example of "smart", environmentally triggered release is shown in Fig. 4a-b: the release rate of the anti-inflammatory drug, piroxicam (PX), and of the negatively charged fluorescent sodium salt (FL) from polypyrrole (PPy) nanoparticles is directly proportional to $\mathrm{pH}$ (Fig. 4a), whereas that of the positively charged rhodamine 6G (R6G) is inversely proportional to pH (Fig. 4b). In Fig. 4c, gentamicin release is shown to be triggered in the event of infection and the associated increase in the acidity of the environment around the carrier. Such systems are attractive because of their potential to obliterate the need for the prophylactic use of antibiotics and at the same time minimize the systemic side effects. Also, the intracellular environment has a markedly higher redox potential than the extracellular milieu, with the concentration of glutathionate inside the cell $(\sim 10 \mathrm{mM})$ being three orders of magnitude higher than that outside the cell $(\sim 10 \mu \mathrm{M})$. The cytosolic cleavage of disulfide or thioester bonds can be used as a trigger for the controlled release of therapeutics, and one example is shown in Fig. 4d: as the concentration of dithiothreitol, which cleaves the disulfide bond, is increased, so does the rate of release of SN-38 from 2,6-bis(hydroxymethyl)aniline based polymeric nanoparticles increase too. The concentration of an enzyme in the environment surrounding the carrier is another parameter that could control the drug release rate: an example comes from the concentration of $\beta$-Dgalactosidase being directly proportional to the rate of release of nitric oxide from the 
enzymatically cleaved O2-( $\beta$-D-galactopyranosyl)1-(N-isopropylamino)diazen-1-ium-1,2diolate (Gal-IPA/NO) prodrug[28]

2.4.2. Carriers stimulated by the external physical signal-A special class of materials capable of having the release triggered by a change in a specific environmental parameter are those in which such a change is being implemented not spontaneously, by a biological process, but by the clinical operator. In such a case, the material responds to an optical, magnetic, thermal or acoustic signal from an external device. PPy scaffolds, for example, can exhibit tunable release by controlling the magnitude of electrical stimulation applied to the polymer, which causes an increase in the interplanar spacing in the inverse opal structure of PPy and the release of the intercalated drug [32]. Calcium alginate hydrogels also enable the tunability of the drug release by controlling the electric field strength. In one study, the release of folic acid increased from 13 to 18 to 25 to $31 \%$ after the first $30 \mathrm{~min}$ of immersion as the electric field strength increased from 0 to 0.5 to 1 to 5 V[33]. The release of Texas-red-conjugated bovine serum albumin (BSA) from gelatinous microspheres incorporating thermoresponsive poly( $\mathrm{N}$-isopropylacrylamide-co-acrylamide) (PNIPAm-co-AAm) can be tuned by controlling the degree of deswelling, which itself is controllable by temperature: from 20 to $100 \%$ after $30 \mathrm{~min}$ as the temperature increased from 22 to $42{ }^{\circ} \mathrm{C}[34]$. The drug release with thermoresponsive polymers need not always increase with temperature, as exemplified by the release of fluorescent 1-

anilinonaphthalene-8-sulfonic acid from polystyrene-block-poly(N-isopropylacrylamide)block-polystyrene (PS-b-PNIPAm-bPS) nanoparticles decreasing from $10-15 \%$ after $3.6 \mathrm{~h}$ at $25{ }^{\circ} \mathrm{C}$ to $4-8 \%$ after the same time at $45{ }^{\circ} \mathrm{C}$, i.e., above the cloud point temperature of the PNIPAm[35].

In a nanomachine domain, the concentration of Nile blue was made precisely tunable by varying the rotation speed of nanoparticles in a nanomotor composed of a plasmonic, trilayered $\mathrm{Au} / \mathrm{Ni} / \mathrm{Au}$ nanorod as a rotor, a $200 \mathrm{~nm}$ thick magnetic film of $\mathrm{Au} / \mathrm{Ni} / \mathrm{Cr}$ as the bearing and microelectrodes as the stator [36]. Specifically, the release rate of Nile blue bound to the surface of plasmonic nanorods by incubation in a solution monotonically increased with the rotation speed of the nanorods. This speed was controllable using the electric voltage applied to the quadruple microelectrodes in the device.

Then, a continuous increase in the magnetic field intensity in the $0-250 \mathrm{mT}$ range linearly increased the amount of released docetaxel from a battery-less MEMS device composed of a drug reservoir wrapped up in a magnetic membrane made up of iron oxide nanoparticles dispersed in a polydimethylsiloxane (PDMS) matrix[37]. Ultrasound irradiation has not been controlled in intensity so as to produce tunable release profiles, but the use of on/off modulation of the field to control dosing was noted in the literature: for example, the release of rhodamine B from polystyrene-PDMS block co-polymeric nanofibers would be halted without the field and induced at a constant rate when the field is switched on[38]. Similar on/off modulations of photothermal, continuous-wave near-infrared laser irradiation allowed a precise control of the amount of released doxorubicin from mesoporous copper sulfide nanoparticles capped with hyaluronic acid to be achieved[39]. 


\subsection{Examples of intrinsically tunable carriers}

A limited number of intrinsic structural parameters has been used to control drug release rates in a tunable fashion. For example, porosity can be tuned to control the drug release; one example comes from a study in which an increase in the pore size from 15 to 43 to 95 $\mathrm{nm}$ in an oxidized porous silicon carrier gradually increased the release rate of daunorubicin (Fig. 5c) [40]. Similarly, the increase of the pore size of hollow silicon microspheres from 15 to 25 to $50 \mathrm{~nm}$ entailed a directly proportional increase in the release rate of quantum dots from these microspheres [41]. Control over the current density and anodization time in the $40-60 \mathrm{~mA} / \mathrm{cm}^{2}$ and $5-10 \mathrm{~s}$ range allowed for tuning the porosity of mesoporous silicon from 53 to 60 to $72 \%$ and the corresponding release of a-chymotrypsinogen from 3 to 45 to $85 \%$ after $24 \mathrm{~h} \mathrm{[42].}$

The crosslinking ratio in a polymer, typically controlled with the molar ratio between the monomer and the cross-linker in the synthesis stage, has been frequently used to control the diffusion coefficient of the drug entrapped within the polymer matrix and the rate of its release into the environment. As for the tunable effect, increasing the crosslinking ratio of calcium alginate in the $0.3-1.3$ range proportionally decreased the amount of released folic acid[33]. Then, increasing the crosslinking ratio of gelatin in starch/gelatin microspheres from 0 to 12.5 to $25 \%$ using glutaraldehyde decreased the released amount of methylene blue from 99 to 70 to $68 \%$ after $24 \mathrm{~h}$ [43]. Also, controlling the concentration of the crosslinker, 3,3'-ethylidene-bis(N-vinyl-2-pyrrolidone) (EBVP), in the $0.15-0.20 \%$ range allowed the release of niacin to be made tunable to anywhere between 5 and $20 \mathrm{~h}$ [44]. Interestingly, no changes in crosslinking outside this range had an effect on the drug release, illustrating the limitations of this type of drug release control. In fact, it appears that crosslinking degree ranges in which the tunable release effect is achievable are often narrow and different depending on whether the release is short- or long-term. For example, increasing the crosslinking degree in gelatin scaffolds from 0 to $23.7 \%$ had no long-term ( 7 - 21 days) effect on the release of mitomycin $\mathrm{C}$, even though it did have a threefold effect on the decrease of the released amount after $24 \mathrm{~h}$ [45]. Finally, although the crosslinking degree usually inversely relates to the water ingression and the drug diffusion and release rates, opposite effects are frequently found in the literature. Thus, because the concentration of active norbornene and thiol sites that bind the drug dropped with crosslinking, an increase in the molecular weight of a poly(ethylene glycol) (PEG) hydrogel led to an increase in the release rate of BSA: 40 and $60 \%$ after 7 days for 2.5 and $4 \mathrm{kDa}$, respectively[46]. The same effect explains why an increase in the crosslinking density of both chain- and step-grown PEG caused a monotonous increase in the rate of release of BSA[47].

Crosslinking can be the key parameter for tuning the release rate of drugs not only from hydrogels, but from vitreous and elastomeric polymers too. For example, an increased acrylation of trimethylene carbonate (TMC) elastomer and the corresponding decrease in the ratio between TMC and D,L-lactide (DLLA) in their copolymer from 100:0 to 50:50, indicating a more efficient crosslinking reaction, gradually increased the release of BSA from 0 to 13 to 20 to $35 \%$ after 40 days of the release time[48]. In this case, the fundamental understanding of the process is technically easier because the rate-limiting step is usually diffusion and occasionally osmosis, given that the effects of polymer swelling and 
erosion have a lesser effect on the release kinetics than in hydrogels. Diffusion of the solute through the polymeric matrix is, of course, dependent on a plenty of other parameters, many of which affect or are indirectly affected by crosslinking, including porosity, tortuosity, pore connectivity, the hydrodynamic radius of the solute, hydrophobicity and polarity of the polymer relative to the solute, the potential for hydrogen or van de Waals bonding between the solute and the polymer, solute concentration gradient as the driving force for diffusion, distribution of the repeat units in a polymer, geometry of the polymeric implant, crystallinity, end group character and identity, chain defects and rigidity, et cetera; however, not all of them are readily tunable in a sense in which this term is being used in this study.

The characteristic U-shaped degradation profile of PLGA as a function of LA/GA ratio[49] has been frequently used to tune the release rate of drugs entrapped in PLGA as a function of the weight ratio of these two monomers [50]. The release of thyrotropin releasing hormone from PLGA microspheres increased with the increase in the GA content of the polymer in the LA/GA ratio range from 50/50 to 100/0 [27]. Varying the LA/GA ratio from $70 / 30$ to $90 / 10$ while keeping other parameters constant decreased mitomycin-C release from 70 to $30 \mathrm{wt} . \%$ after three months of release [52]. Likewise, lowering the feed ratio between acrylamide (AAm) and (r)-a-acryloyloxy- $\beta, \beta$-dimethyl- $\gamma$-butyrolactone (DBA) in a thermoresponsive copolymer decreased the rate of release of dexamethasone from $100 \%$ after a week for $\mathrm{AAm} / \mathrm{DBA}=5.25$ to $15 \%$ for $\mathrm{AAm} / \mathrm{DBA}=0.9[53]$. The drug release from electrospun polymeric fibers composed of polycaprolactone (PCL) and PLGA was also made tunable by controlling the weight ratio between the two polymeric components [54]. Specifically, anti-HIV drug Tenofovir disoproxil was released faster at larger PCL contents and more sustainably at larger PLGA contents. Also, micelles made of the block copolymer composed of poly(ethylene glycol)-b-poly(lactic acid) (PEG-b-PLA) and PLA-b-PNIPAm displayed a reduced release of ibuprofen as the content of PLA-b-PNIPAm increased [55]. Tuning the release as a function of the weight ratio between the two components of a carrier is often the consequence of their different affinity for the drug, and one example comes from the tunability of the release of recombinant human insulin-like growth factor 1 (rhIGF-1): with rhIGF-1 binding more intensely to more biodegradable kerateine (KTN) than to less biodegradable keratose (KOS), increasing the KTN/KOS weight ratio in a polymer increased the rate of release of rhIGF-1[56]. The release was tunable within the $10-70 \%$ range after 7 days, as defined by the release from pure KTN (10\%) and pure KOS (70\%), respectively.

Still, although polymeric microspheres have been used as drug delivery carriers since the 1980s [58], the effects other than variations in the bulk composition and crosslinking ratio have been rarely used for tunable release purposes. Controlling the thickness of the polymeric film deposited over a drug reservoir can produce finely tuned release profiles [59]; however, this concept has been rarely ever translated to polymeric particle systems. Yet, there are notable examples. For example, hollow composite gold/polymeric microcapsules were 3D printed and the variation in the weight ratio of PLGA in the precursor solution from 0.5 to $5 \mathrm{wt} . \%$ controlled the capsule thickness and allowed the release of horseradish peroxidase to be tuned to profiles shown in Fig. 5d. Also, hybrid $\mathrm{PLGA} / \mathrm{TiO}_{2}$ microspheres were fabricated using droplet-based microfluidics [60] and their surface texture was varied by changing the mass ratio between titanium tetraisopropoxide (TTIP) and PLGA in the dispersed phase. The higher the content of TTIP, the deeper the wrinkles and the faster the 
drug release, a phenomenon which was attributed to a larger specific surface area leading to a more rapid release of Tanshinone IIA as the drug. The degree of oxidation of carboxymethyl cellulose microspheres with periodate was used to increase the release of doxorubicin from 15 to 20 to $25 \%$ as the oxidation degree increased from 9 to 17 to $35 \%$, respectively[61]. The use of multiple other, finer polymer microparticle properties, - e.g., surface texture and chemistry, core/shell size/thickness, self-assembly features, etc. - is expected to open up a whole new avenue in controlled drug release research.

Other effects are also noted in the literature. Changing the degree of substitution of deoxycholic acid in nanoparticles based on hyaluronic and deoxycholic acid conjugates (HA-DOCA) from 5.9 to 7.6 to 9.4 changed the released amount of doxorubicin from 49 to 58 to $70 \%$ [62]. Changing the particle size of magnesium carbonate from $25-50$ to $75-$ 100 to $>200 \mu \mathrm{m}$ on average decreased the amount of released ibuprofen after $24 \mathrm{~h}$, albeit within a very narrow range, from 98 to 93 to $87 \%$ [63]. The effect of the drug on the structure of the carrier is never to be underestimated either, as the result of which the concentration of the drug loaded into the carrier can often be used as a parameter to produce tunable release profiles. One example comes from the increase in the released percentage of the loaded dexamethasone paralleling an increase in the amount of the drug present in PCL/ silk fibroin nanoparticles as the carrier[64]. The release rate of doxorubicin from liposomes similarly increased in parallel with the increase in the concentration of the encapsulated drug and was made tunable using drug-to-lipid ratio as the control parameter[65]. The opposite effect was observed in the case of loading HA-DOCA nanoparticles with different amounts of doxorubicin: as the content of the drug in the carrier increased, the cumulative rate of the release of the drug decreased ( 71 vs. 42 vs. $32 \%$ after $24 \mathrm{~h}$ for 5,10 and 20 wt.\% loadings, respectively)[62]. Such a disparity between the effects of technically identical parameters illustrates the complexity of understanding and controlling the mechanisms of drug release in general. Tunability achievable in a system with a specific drug/carrier combination may be obliterated when the identity of the drug changes. One example comes from the aforementioned tunable release of rhIGF-1 from KOS/KTN polymer, impossible to repeat with another growth factor, recombinant human bone morphogenetic protein 2, or with an antibiotic, ciprofloxacin[56]. Finally, Table 2 lists some of the patented carriers with intrinsically tunable release properties. Table 3 provides a summary of the carriers referred to in the text and of their categorization based on two major groups - intrinsic vs. extrinsic and two subgroups: tunable vs. tailorable and smart vs. manual, respectively (Fig. 2).

\section{Expert Opinion}

A central problem in the elucidation of parameters that could be used as "magic knobs" and allow the drug release to be made intrinsically tunable is the inevitable entanglement of variables defining the microstructure of the carrier. Changing one of these parameters is bound to have an effect on other parameters, which may affect the release in an uncontrolled manner and deter the tunable effect. For example, increasing the size of sucrose acetate isobutyrate microsphere depots at first decreased the release rate of risperidone because of a lesser particle surface area conducive to drug diffusion, but then increased it at even larger particle sizes because of the larger porosity of the large particles[77]. Increasing the particle size also does not only lower the specific surface area of the material, but also lowers the 
particle curvature and, thus, the surface energy, affecting particle-particle interaction, surface binding and release of the drug, and the diffusion of the drug across the particle/solution interface. Thus, although increased surface area of a flat surface directly translates to increased released rate[78], increased surface area in a particulate system comes at the cost of increased surface tension and compaction of the crystalline order at the particle surface, which can act as a barrier to the release. Still, the medical need for such carriers is obvious and its fueling the drive for the discovery is expected to overcome these fundamental challenges and ambiguities. The ease, the elegance and the practical appeal of using a single parameter to control the release rate as opposed to compromising between a multitude thereof will also be a crucial contributor to the development of a new generation of intrinsically tunable release carriers.

Much has been said about the necessity for the input of materials scientists on devising more advanced drug delivery platforms. However, the ability to control and theoretically predict physicochemical properties of materials in a tunable manner still greatly surpasses the ability to control and model their drug release properties. Insufficient versatility and inferior stimuli-response sophistication of drug delivery devices compared to those of electronic devices, the majority of which owe their function to the tunability of one or more of their components, speaks in favor of this disparity. For example, today we know that nanomaterials exhibit a markedly broader variety of potential properties than their bulk counterparts, and tunability is one of their hallmarks. Owing to the quantum confinement effect, the size of semiconducting particles at the nanoscale can be controlled to tune their bandgap and, thus, the photon emission frequency to the desired value[79]. Simple variations of graphene nanoribbon width, density or the doping level also allow for the tunability of the bandgap and the corresponding metal-oxide-semiconductor field-effect transistor properties[80][81][82]. Albeit having an insignificant effect in many other 2D materials, including $\mathrm{MoS}_{2}$, a precise control over the number of deposited monoatomic layers of phosphorene gives a precise control over the magnitude of the transport gap of the material[83]. Plasmon resonance in gold nanostructures could be tuned by varying the particle diameter[84], aspect ratio[85], thickness in a layer[86], et cetera. Sophisticated superstructures, such as photonic crystals and metamaterials, also allow for the tunability of optical properties through a control over their geometric features and translational symmetry[87][88]. An unexplored, albeit bold question is if the tunability that typifies the quantum properties of nanoparticles could be translated to the tunability of drug release profiles.

Magnetic ceramics are instructive in terms of the intrinsic tunability of properties. By controlling the distribution of cations at octahedral and tetrahedral sites of the spinel lattice of ferrites, more than one of their magnetic properties, ranging from susceptibility to permeability to Neel temperature to coercivity to remanence, can be tuned[89]. Variation of the stoichiometric ratio between a couple of metallic dopants at the A site of the $\mathrm{ABO}_{3}$ perovskite structure of nanostructured manganites, e.g., $x$ in $\mathrm{La}_{1-x} \mathrm{Sr}_{\mathrm{x}} \mathrm{MnO}_{3+\delta}$, can be employed to vary their Curie temperature and adjust it to the hyperthermically or thermoblastically targeted tissue in magnetic cancer therapies[90]. Controlling a similar stoichiometric parameter $x$, though defining $\mathrm{Co} / \mathrm{Ni}$ molar ratio in magneto-dielectric ferrite, $\mathrm{BaSrCo}_{2-\mathrm{x}} \mathrm{Ni}_{\mathrm{X}} \mathrm{Fe}_{12} \mathrm{O}_{22}$, allows for the simultaneous tuning of the narrowband reflection 
reduction in a wide microwave frequency range at low $x$ and high Co contents and of the wideband transmittance attenuation in a similarly wide frequency range at high $x$ and high $\mathrm{Ni}$ contents[91]. Atomic layer engineering has also enabled the tuning of an array of properties and transitions in alloys and ceramics depending on the atomically precise doping levels and deposited geometries, ranging from 1D ribbons and strings to nanoladders to sheets and films with an atomically defined thickness[92][93]. The question is whether similar materials, methods and structural effects could be employed to yield tunable release profiles too.

This question may be the starting point for the expansion of the repertoire of materials with tunable release properties. Traditionally, polymers have been the main choice for tunable release agents because of the ease with which this control be intrinsically achieved using parameters such as monomeric unit ratio, crosslinking ratio, etc. Until recently, it was thought that calcium phosphates, like most ceramics, did not have the potential for controllable release, let alone tunable. However, this was refuted with the development of self-setting pastes in which the release of antibiotics could be modeled with simplistic equations allowing for the prediction of release properties depending on a single, easily controllable compositional parameter[22]. As far as the externally triggered release platforms are concerned, piezoelectric and magnetostrictive materials, deforming in direct proportionality with the intensity of the applied electric[94] or magnetic[95] fields, respectively, and potentially releasing the captured drug in a kinetically controllable fashion, present another class of ceramics that could be investigated for tunable delivery. Nanotubes, e.g. boron nitride[96], or composites with polymers[97] present particularly attractive subclasses of such materials owing to their proven drug loading and release capabilities. Electrically responsive polymers[98] will also offer a whole new avenue in the utilization of the proposed effects. Therefore, it is important that research aiming to discover new materials for controlled release continues. Along the way, therapeutic synergies emerging from the carrier properties other than those enabling the tunable release are expected to be derivable by serendipity, if not by design.

One such diversification of the types of materials capable of exhibiting tunable release may go hand-in-hand with the distancing from the now classical tissue engineering paradigm of devising platforms that would regenerate, but not augment the replaced tissues. Thinking in this bold direction and engaging in the use of materials whose therapeutic effects may be due to their moderate toxicity and invasiveness of biophysical structures (every therapy is conditioned upon the creation of a wound) may bring us closer to other advanced concepts in the intrinsically tunable drug delivery. One of them would be the use of particle aggregation degrees through the control of surface chemistry and charge. Another direction worth exploring belongs to the exploitation of transitory, metastable, non-equilibrium states for the kinetic control of release patterns. Polymorphic transitions have been used to mediate drug release [99], but no tunable effects have been reported so far. Particle shell-to-core volume ratio is another parameter that has been used to produce different release profiles; however, the correlation between this parameter and release rate was rather volatile, not allowing for the tunable effect [100]. 
Finally, although the division to extrinsic/intrinsic and tunable/tailorable release carriers was introduced to clarify the perspective on them, it is expected that, as ever, conditions for the creation of the most prolific systems would be set on the grounds where the boundaries crush and multiple concepts merge into one.

\section{Acknowledgments}

Acknowledged for support is NIH grant R00-DE021416: Osteogenic Calcium Phosphate Nanoparticles with Designable Drug Release Kinetics. The authors also acknowledge Victoria Wu of UIC for drawing Fig. 2.

\section{References}

1*. Uskoković V. When 1+1> 2: Nanostructured composites for hard tissue engineering applications. Mater Sci Eng C. 2015; 57:434-51. A critical review piece highlighting the need for "smart, multicomponent systems capable of delivering therapeutic payloads at the ideal location by means of the right targeting agents and at the ideal time points through release triggered by an in situ detection of disease markers".

2*. Zhang Y, Chan HF, Leong KW. Advanced materials and processing for drug delivery: the past and the future. Adv Drug Deliv Rev. 2013; 65:104-20. A review of FDA-approved drug delivery systems and materials in the exploratory stage for the same application: block polymers, polymer-drug conjugates, recombinant proteins and smart systems, alongside the most common fabrication techniques. [PubMed: 23088863]

3. Rau JV, Antoniac I, Cama G, et al. Bioactive Materials for Bone Tissue Engineering. Biomed Res Int. 2016; 2016:3741428. [PubMed: 26942191]

4. Einhorn TA, Gerstenfeld LC. Fracture healing: mechanisms and interventions. Nat Rev Rheumatol. 2015; 11:45-54. [PubMed: 25266456]

5. O'Loughlin, A.; O'Brien, T. Topical stem and progenitor cell therapy for diabetic foot ulcers. INTECH Open Access Publisher; 2011.

6. Tan DT, Chee SP, Lim L, et al. Randomized clinical trial of a new dexamethasone delivery system (Surodex) for treatment of post-cataract surgery inflammation. Ophthalmology. 1999; 106:223-31. [PubMed: 9951469]

7. Hadayer A, Schaal S. Delivery of steroids into the eye for the treatment of macular edema. Expert Opin Drug Deliv. 2016:1-9.

8. Dang W, Daviau T, Ying P, et al. Effects of GLIADEL® wafer initial molecular weight on the erosion of wafer and release of BCNU. J Control Release. 1996; 42:83-92.

9. Parmar H, Lightman SL, Allen L, et al. Randomised controlled study of orchidectomy vs longacting D-Trp-6-LHRH microcapsules in advanced prostatic carcinoma. Lancet. 1985; 326:1201-5.

10. Benagiano G, Gabelnick H, Farris M. Contraceptive devices: subcutaneous delivery systems. Expert Rev Med Devices. 2008; 5:623-37. [PubMed: 18803473]

11. Gambling D, Hughes T, Martin G, et al. A comparison of Depodur ${ }^{\mathrm{TM}}$, a novel, single-dose extended-release epidural morphine, with standard epidural morphine for pain relief after lower abdominal surgery. Anesth Analg. 2005; 100:1065-74. [PubMed: 15781524]

12. Gefvert O, Eriksson B, Persson P, et al. Pharmacokinetics and D2 receptor occupancy of longacting injectable risperidone (Risperdal Consta ${ }^{\mathrm{TM}}$ ) in patients with schizophrenia. Int $\mathrm{J}$ Neuropsychopharmacol. 2005; 8:27-36. [PubMed: 15710053]

13. James IGV, O'Brien CM, McDonald CJ. A randomized, double-blind, double-dummy comparison of the efficacy and tolerability of low-dose transdermal buprenorphine (BuTrans ${ }^{\circledR}$ seven-day patches) with buprenorphine sublingual tablets (Temgesic ${ }^{\circledR}$ ) in patients with osteoarthritis pain. J Pain Symptom Manage. 2010; 40:266-78. [PubMed: 20541900]

14. Syed YY, Keating GM. Extended-release intramuscular naltrexone (VIVITROL®): a review of its use in the prevention of relapse to opioid dependence in detoxified patients. CNS Drugs. 2013; 27:851-61. [PubMed: 24018540] 
15. Dhillon B, Kamal A, Leen C. Intravitreal sustained-release ganciclovir implantation to control cytomegalovirus retinitis in AIDS. Int J STD AIDS. 1998; 9:227-30. [PubMed: 9598751]

16. Schurman DJ, Trindade C, Hirshman HP, et al. Antibiotic-acrylic bone cement composites. Studies of gentamicin and Palacos. J Bone Jt Surg Am. 1978; 60:978-84.

17. Lewis G, Janna $\mathrm{S}$. The in vitro elution of gentamicin sulfate from a commercially available gentamicin-loaded acrylic bone cement, VersaBond ${ }^{\mathrm{TM}}$ AB. J Biomed Mater Res Part B Appl Biomater. 2004; 71:77-83. [PubMed: 15368230]

18. Talan DA, Naber KG, Palou J, et al. Extended-release ciprofloxacin (Cipro XR) for treatment of urinary tract infections. Int J Antimicrob Agents. 2004; 23:54-66.

19. Carvalho B, Riley E, Cohen SE, et al. Single-dose, sustained-release epidural morphine in the management of postoperative pain after elective cesarean delivery: results of a multicenter randomized controlled study. Anesth Analg. 2005; 100:1150-8. [PubMed: 15781537]

20. Imaz I, Maspoch D, Rodríguez-Blanco C, et al. Valence-Tautomeric Metal-Organic Nanoparticles. Angew Chemie. 2008; 120:1883-6.

$21 *$. Uskoković V, Desai TA. Phase composition control of calcium phosphate nanoparticles for tunable drug delivery kinetics and treatment of osteomyelitis. I. Preparation and drug release. J Biomed Mater Res Part A. 2013; 101:1416-26. A study showing that the stoichiometry of calcium phosphates could be used to tailor their degradability and the corresponding release profiles of fluorescein and BSA to desired degradation and release timescales.

$22 * *$. Ghosh S, Wu V, Pernal S, et al. Self-setting calcium phosphate cements with tunable antibiotic release rates for advanced antimicrobial applications. ACS Appl Mater Interfaces. 2016; 8:7691708. A study demonstrating that the weight ratio between two calcium phosphate components of a self-setting paste could be used as a control parameter in tuning the release of different antibiotics to anywhere between 0 and 14 days. Also the first study to show that the kinetics of a phase transition within the carrier could be controlled to create tunable release profiles. [PubMed: 26958867]

23. Maver T, Hribernik S, Mohan T, et al. Functional wound dressing materials with highly tunable drug release properties. RSC Adv. 2015; 5:77873-84.

24. Muhsin MDA, George G, Beagley K, et al. Effects of chemical conjugation of L-leucine to chitosan on dispersibility and controlled release of drug from a nanoparticulate dry powder inhaler formulation. Mol Pharm. 2016; 13:1455-66. [PubMed: 26998555]

25. Roy A, Jhunjhunwala S, Bayer E, et al. Porous calcium phosphate-poly (lactic-co-glycolic) acid composite bone cement: A viable tunable drug delivery system. Mater Sci Eng C. 2016; 59:92101.

26. Parker J, Mitrousis N, Shoichet MS. Hydrogel for Simultaneous Tunable Growth Factor Delivery and Enhanced Viability of Encapsulated Cells in Vitro. Biomacromolecules. 2016; 17:476-84. [PubMed: 26762290]

27. Coburn JM, Na E, Kaplan DL. Modulation of vincristine and doxorubicin binding and release from silk films. J Control Release. 2015; 220:229-38. [PubMed: 26500149]

28. Holland RJ, Paulisch R, Cao Z, et al. Enzymatic generation of the NO/HNO-releasing IPA/NO anion at controlled rates in physiological media using $\beta$-galactosidase. Nitric Oxide. 2013; 35:131-6. [PubMed: 24126017]

29*. Samanta D, Meiser JL, Zare RN. Polypyrrole nanoparticles for tunable, pH-sensitive and sustained drug release. Nanoscale. 2015; 7:9497-504. A study on a composite containing calcium alginate hydrogel and polypyrrole nanoparticles with tunable release profiles thanks to the ability of polypyrrole to respond to the alkalinity of its chemical environment. [PubMed: 25931037]

30. Pichavant L, Amador G, Jacqueline C, et al. pH-controlled delivery of gentamicin sulfate from orthopedic devices preventing nosocomial infections. J Control Release. 2012; 162:373-81. [PubMed: 22771533]

31. Cai K, Yen J, Yin Q, et al. Redox-responsive self-assembled chain-shattering polymeric therapeutics. Biomater Sci. 2015; 3:1061-5. [PubMed: 26146551]

32*. Sharma M, Waterhouse GIN, Loader SWC, et al. High surface area polypyrrole scaffolds for tunable drug delivery. Int J Pharm. 2013; 443:163-8. A study on polypyrrole inverse opal thin 
films with tunable release profiles thanks to the ability of polypyrrole to respond to electrical stimulation. [PubMed: 23318368]

33. Paradee N, Sirivat A, Niamlang S, et al. Effects of crosslinking ratio, model drugs, and electric field strength on electrically controlled release for alginate-based hydrogel. J Mater Sci Mater Med. 2012; 23:999-1010. [PubMed: 22354328]

34. Sung B, Kim C, Kim MH. Biodegradable colloidal microgels with tunable thermosensitive volume phase transitions for controllable drug delivery. J Colloid Interface Sci. 2015; 450:26-33. [PubMed: 25797395]

35. Rahikkala A, Aseyev V, Tenhu H, et al. Thermoresponsive Nanoparticles of Self-Assembled Block Copolymers as Potential Carriers for Drug Delivery and Diagnostics. Biomacromolecules. 2015; 16:2750-6. [PubMed: 26207325]

36**. Xu X, Kim K, Fan D. Tunable Release of Multiplex Biochemicals by Plasmonically Active Rotary Nanomotors. Angew Chemie Int Ed. 2015; 54:2525-9. A study reporting on the design of a nanomotor in which the rotation speed of nanoparticles, controllable using the electric voltage applied to the quadruple microelectrodes in the device, was the control parameter for tuning the drug release.

37**. Pirmoradi FN, Jackson JK, Burt HM, et al. On-demand controlled release of docetaxel from a battery-less MEMS drug delivery device. Lab Chip. 2011; 11:2744-52. A study showing that the magnetic field strength in an MEMS drug delivery device monotonously relates to the rate of release of docetaxel. [PubMed: 21698338]

38. Demirel GB, Buyukserin F, Morris MA, et al. Nanoporous polymeric nanofibers based on selectively etched PS-b-PDMS block copolymers. ACS Appl Mater Interfaces. 2011; 4:280-5. [PubMed: 22107361]

39**. Feng Q, Zhang Y, Zhang W, et al. Tumor-targeted and multi-stimuli responsive drug delivery system for near-infrared light induced chemo-phototherapy and photoacoustic tomography. Acta Biomater. 2016 A study on the application of modulated photothermal radiation to control the amount of released doxorubicin from hyaluronan-capped $\mathrm{Cu}_{2} \mathrm{~S}$ nanoparticles.

40. Hou H, Nieto A, Ma F, et al. Tunable sustained intravitreal drug delivery system for daunorubicin using oxidized porous silicon. J Control Release. 2014; 178:46-54. [PubMed: 24424270]

41. Martinez JO, Chiappini C, Ziemys A, et al. Engineering multi-stage nanovectors for controlled degradation and tunable release kinetics. Biomaterials. 2013; 34:8469-77. [PubMed: 23911070]

42. Pastor EL, Reguera-Nuñez E, Matveeva E, et al. Pore size is a critical parameter for obtaining sustained protein release from electrochemically synthesized mesoporous silicon microparticles. PeerJ. 2015; 3:e1277. [PubMed: 26557423]

43. Phromsopha T, Baimark Y. Preparation of starch/gelatin blend microparticles by a water-in-oil emulsion method for controlled release drug delivery. Int J Biomater. 2014; 2014

44. Sirc J, Hrib J, Vetrik M, et al. The Use of a Hydrogel Matrix for Controlled Delivery of Niacin to the Gastrointestinal Tract for Treatment of Hyperlipidemia. Physiol Res. 2015; 64:S51-60. [PubMed: 26447595]

45*. Zhu J, Yang F, He F, et al. A tubular gelatin scaffold capable of the time-dependent controlled release of epidermal growth factor and mitomycin C. Colloids Surfaces B Biointerfaces. 2015; 135:416-24. A study illustrating the limitations in the use of the crosslinking degree to tune the release of mitomycin C from gelatin scaffolds. [PubMed: 26277717]

46. Lee S, Tong X, Yang F. Effects of the poly (ethylene glycol) hydrogel crosslinking mechanism on protein release. Biomater Sci. 2016; 4:405-11. [PubMed: 26539660]

47. Lee S, Tong X, Yang F. The effects of varying poly (ethylene glycol) hydrogel crosslinking density and the crosslinking mechanism on protein accumulation in three-dimensional hydrogels. Acta Biomater. 2014; 10:4167-74. [PubMed: 24887284]

48. Chapanian R, Amsden BG. Osmotically driven protein release from photo-cross-linked elastomers of poly (trimethylene carbonate) and poly (trimethylene carbonate-co-D, L-lactide). Eur J Pharm Biopharm. 2010; 74:172-83. [PubMed: 19948219]

49. Cameron, RE.; Kamvari-Moghaddam, A. Rate Bioresorbable Mater. Cambridge Engl: Woodhead Pubishing Ltd; 2008. Synthetic bioresorbable polymers, Degrad; p. 43-66. 
50. Alexis F. Factors affecting the degradation and drug-release mechanism of poly(lactic acid) and poly [(lactic acid)-co-(glycolic acid)]. Polym Int. 2005; 54:36-46.

51. Toshiro H, Hiroaki O, Yasuaki O, et al. Factors influencing the profiles of TRH release from copoly (d, l-lactic/glycolic acid) microspheres. Int J Pharm. 1991; 72:199-205.

52. Gumuşderelioğlu M, Deniz G. Sustained release of mitomycin-C from poly (DL-lactide)/poly (DL-lactide-co-glycolide) films. J Biomater Sci Polym Ed. 2000; 11:1039-50. [PubMed: 11211156]

53. Prosperi-Porta G, Muirhead B, Sheardown H. Tunable release of ophthalmic therapeutics from injectable, resorbable, thermoresponsive copolymer scaffolds. J Biomed Mater Res Part B Appl Biomater. 2015

54. Carson D, Jiang Y, Woodrow KA. Tunable Release of Multiclass Anti-HIV Drugs that are WaterSoluble and Loaded at High Drug Content in Polyester Blended Electrospun Fibers. Pharm Res. 2016; 33:125-36. [PubMed: 26286184]

55*. Wu C, Ma R, He H, et al. Fabrication of complex micelles with tunable shell for application in controlled drug release. Macromol Biosci. 2009; 9:1185-93. A study demonstrating how the weight ratio between two polymeric components in a block copolymer can be used to tune the drug release rate. [PubMed: 19842158]

56. Ham TR, Lee RT, Han S, et al. Tunable Keratin Hydrogels for Controlled Erosion and Growth Factor Delivery. Biomacromolecules. 2015; 17:225-36. [PubMed: 26636618]

57*. Gupta MK, Meng F, Johnson BN, et al. 3D printed programmable release capsules. Nano Lett. 2015; 15:5321-9. A study showing a tremendous difference in the release of horseradish peroxidase from gold-nanorod/PLGA microcapsules as a function of the variation of the microcapsule shell thickness. [PubMed: 26042472]

58. Freiberg S, Zhu XX. Polymer microspheres for controlled drug release. Int J Pharm. 2004; 282:118. [PubMed: 15336378]

59. Lance KD, Good SD, Mendes TS, et al. In Vitro and In Vivo Sustained Zero-Order Delivery of Rapamycin (Sirolimus) From a Biodegradable Intraocular DeviceSustained Zero-Order Delivery of Rapamycin. Invest Ophthalmol Vis Sci. 2015; 56:7331-7. [PubMed: 26559479]

60. Dong H, Tang G, Ma T, et al. One-step fabrication of inorganic/organic hybrid microspheres with tunable surface texture for controlled drug release application. J Mater Sci Mater Med. 2016; 27:1-8. [PubMed: 26610924]

61. Weng L, Rostamzadeh P, Nooryshokry N, et al. In vitro and in vivo evaluation of biodegradable embolic microspheres with tunable anticancer drug release. Acta Biomater. 2013; 9:6823-33. [PubMed: 23419554]

62. Wei WH, Dong XM, Liu CG. In vitro investigation of self-assembled nanoparticles based on hyaluronic acid-deoxycholic acid conjugates for controlled release doxorubicin: effect of degree of substitution of deoxycholic acid. Int J Mol Sci. 2015; 16:7195-209. [PubMed: 25837468]

63. Zhang P, Gómez De La Torre TZ, Forsgren J, Bergström C, Strømme M. Diffusion-Controlled Drug Release from the Mesoporous Magnesium Carbonate Upsalite®. J Pharm Sci. 2015

64. Gandhimathi C, Edwin NXH, Jayaraman P, et al. Controlled Release of Dexamethasone in PCL/ Silk Fibroin/Ascorbic Acid Nanoparticles for the Initiation of Adipose Derived Stem Cells into Osteogenesis. J Drug Metab Toxicol. 2015; 5

65**. Luo D, Carter KA, Razi A, et al. Porphyrin-phospholipid liposomes with tunable leakiness. J Control Release. 2015; 220:484-94. A study reporting on the use of drug-to-lipid ratio in liposomes as a control parameter for tuning the release rate of doxorubicin and improving bioavailability and spatial distribution of the drug, resulting in "complete and rapid eradication of subcutaneous tumor xenographs in mice”. [PubMed: 26578438]

66. Aguadisch, LMJ.; Rankin, FS. Pharmaceutical delivery device having a siloxane polymer matrix. US Patent US. 07154755. 1988.

67. Li, J.; Yu, H.; Leong, K. Injectable drug delivery systems with cyclodextrin-polymer based hydrogels. US Patent US. 8003125 B2. 2001.

68. Kamath, KR.; Barry, JJ.; Nott, SH. Polymeric coatings for controlled delivery of active agents. US Patent US. 6335029 B1. 2002. 
69. Schachter, DM.; Zhou, Y. Self-gelling tunable drug delivery system. EP Patent EP. 2076247 A2. 2006.

70. Pacetti, SD.; Stewart, GS.; Gong, VM., et al. Methods of application of coatings composed of hydrophobic, high glass transition polymers with tunable drug release rates. US Patent US. 8562669 B2. 2013.

71. Palasis, M.; Sharma, U.; Pham, Q.; Marini, J. Drug-Loaded Fibers. US Patent US. 20120299223 A1. 2009.

72. Evans, BG.; Evans, DC.; Hogrebe, PC., et al. Controlled release tissue graft combination biomaterials. US Patent US. 8685432 B2. 2014.

73. Shoichet, MS.; Lapitsky, Y.; Wang, Y. Tunable sustained release of a sparingly soluble hydrophobic therapeutic agent from a hydrogel matrix. US Patent US. 9205047 B2. 2015.

74. Rijcken, CJF.; Kruijtzer, JAW.; Van, NCF., et al. Tunable, biodegradable linker molecules for transient conjugation of components in drug delivery systems, and drug delivery systems prepared therewith. WO Patent WO. 2012039602 A1. 2012.

75. Hsu, SL.; Ding, N.; Tang, Y., et al. Methods for modulating the release rate of a drug-coated stent. US Patent US. 8293318 B1. 2012.

76. Simpson PM, Dall GF, Breusch SJ, et al. In vitro elution and mechanical properties of antibioticloaded SmartSet HV and Palacos R acrylic bone cements. Orthopade. 2005; 34:1255-62. [PubMed: 16136337]

77. Lin X, Wang J, Xu Y, et al. Tracking the effect of microspheres size on the drug release from a microsphere/sucrose acetate isobutyrate (SAIB) hybrid depot in vitro and in vivo. Drug Dev Ind Pharm. 2016:1-27.

78. Schlesinger E, Johengen D, Luecke E, et al. Tunable A Biodegradable, Thin-Film Polymer Device as a Long-Acting Implant Delivering Tenofovir Alafenamide Fumarate for HIV Pre-exposure Prophylaxis. Pharm Res. 2016:1-8. [PubMed: 26334501]

79. Lei D, Shen Y, Feng Y, et al. Recent progress in the fields of tuning the band gap of quantum dots. Sci China Technol Sci. 2012; 55:903-12.

80. Son YW, Cohen ML, Louie SG. Energy gaps in graphene nanoribbons. Phys Rev Lett. 2006; 97:216803. [PubMed: 17155765]

81. Lv Y, Chang S, Wang H, et al. Energy gap tunable graphene antidot nanoribbon MOSFET: A uniform multiscale analysis from band structure to transport properties. Carbon N Y. 2016; 101:143-51.

82. Li K, Ma X, Zhang Z, et al. Tunable and angle-insensitive plasmon resonances in graphene ribbon arrays with multispectral diffraction response. J Appl Phys. 2014; 115:104306.

83. Das S, Zhang W, Demarteau M, et al. Tunable transport gap in phosphorene. Nano Lett. 2014; 14:5733-9. [PubMed: 25111042]

84. Qin H, Gao Y, Teng J, et al. Laterally tunable plasmon resonance in confined biatomic-layer Ag nanodisks. Nano Lett. 2010; 10:2961-4. [PubMed: 20614911]

85. Anderson LJE, Payne CM, Zhen Y-R, et al. A tunable plasmon resonance in gold nanobelts. Nano Lett. 2011; 11:5034-7. [PubMed: 21973047]

86. Wu T, Yang S, Li X. Tunable plasmon resonances and enhanced local fields of spherical nanocrescents. J Phys Chem C. 2013; 117:8397-403.

87. Khalkhali TF, Bananej A. Tunable complete photonic band gap in anisotropic photonic crystal slabs with non-circular air holes using liquid crystals. Opt Commun. 2016; 369:79-83.

88. Jia X, Wang X, Meng Q, et al. Tunable multi-band chiral metamaterials based on double-layered asymmetric split ring resonators. Phys E Low-Dimensional Syst Nanostructures. 2016; 81:37-43.

89. Goldman, A. Modern ferrite technology. Springer Science \& Business Media; 2006.

90**. Uskoković V, Košak A, Drofenik M. Silica-coated lanthanum-strontium manganites for hyperthermia treatments. Mater Lett. 2006; 60:2620-2. A study demonstrating that the stoichiometric ratio between $\mathrm{La}^{3+}$ and $\mathrm{Sr}^{2+}$ in biocompatible, $\mathrm{SiO}_{2}$-coated $\mathrm{La}_{1-\mathrm{x}} \mathrm{Sr}_{\mathrm{X}} \mathrm{MnO}_{3+\delta} \mathrm{can}$ be used to tune the Curie point of the material and optimize it for the self-regulating hyperthermia treatment of cancer.

Expert Opin Drug Deliv. Author manuscript; available in PMC 2016 December 16. 
91. Stergiou CA, Litsardakis G. Y-type hexagonal ferrites for microwave absorber and antenna applications. J Magn Magn Mater. 2016; 405:54-61.

92. Božović I, Gozar A, Logvenov G, et al. Insights in high-temperature superconductivity from the study of films and heterostructures synthesized by molecular beam epitaxy. J Supercond Nov Magn. 2009; 22:223-7.

93. Wu J, Bollinger AT, Sun Y, et al. Hall effect in quantum critical charge-cluster glass. Proc Natl Acad Sci. 2016; 113:4284-9. [PubMed: 27044081]

94. Dai Y, Guo W, Zhang Z, et al. Electric-field-induced deformation in boron nitride nanotubes. J Phys D Appl Phys. 2009; 42:85403.

95. Yamaura S, Nakajima T, Satoh T, et al. Magnetostriction of heavily deformed Fe-Co binary alloys prepared by forging and cold rolling. Mater Sci Eng B. 2015; 193:121-9.

96. Ciofani G, Danti S, Genchi GG, et al. Boron Nitride Nanotubes: Biocompatibility and Potential Spill-Over in Nanomedicine. Small. 2013; 9:1672-85. [PubMed: 23423826]

97. McCall WR, Kim K, Heath C, et al. Piezoelectric Nanoparticle-Polymer Composite Foams. ACS Appl Mater Interfaces. 2014; 6:19504-9. [PubMed: 25353687]

98. Marques SM, Rico P, Carvalho I, et al. MC3T3-E1 Cell Response to Ti1-x Ag x and Ag-TiN x Electrodes Deposited on Piezoelectric Poly (vinylidene fluoride) Substrates for Sensor Applications. ACS Appl Mater Interfaces. 2016; 8:4199-207. [PubMed: 26840928]

99. Sergeeva A, Sergeev R, Lengert E, et al. Composite Magnetite and Protein Containing CaCO3 Crystals. External Manipulation and Vaterite $\rightarrow$ Calcite Recrystallization-Mediated Release Performance. ACS Appl Mater Interfaces. 2015; 7:21315-25. [PubMed: 26348458]

100*. Orellana BR, Puleo DA. Tailored sequential drug release from bilayered calcium sulfate composites. Mater Sci Eng C. 2014; 43:243-52. A study demonstrating the ability to control the shell-to-core volume ratio of bilayer simvastatin-PLGA composites and, thus, the kinetics of the drug release. 


\section{Five bulleted summary points}

- $\quad$ The fact that many biological processes take place at precise timescales implies that ideal therapeutic platforms would be capable of achieving a sequential, precisely temporally tuned delivery of the right therapeutic agents.

Etymological clarification of the term "tunable" and its distinction from the term "tailorable" is provided: in the case of intrinsically tunable release, a particular compositional or structural parameter is tuned to give a desired release profile, whereas in the case of intrinsically tailorable release, adding or subtracting different carrier components is used to modify the release profile.

- Tunability can also be achieved extrinsically, being caused by the indiscrete variation of an environmental parameter; included in this category are carriers whose release is controlled by a specific disease marker and carriers whose release is controlled by external effects, e.g., heat, light, magnetic field, ultrasound, mechanical force, and electric current.

- $\quad$ A central problem in the elucidation of parameters that could be used as "magic knobs" that allow the drug release to be made intrinsically tunable is the inevitable entanglement of variables defining the microstructure of the carrier.

Identical parameters often exert diametrically opposite effects on drug release in different carriers, illustrating the complexity of understanding and controlling the mechanisms of drug release in general. 


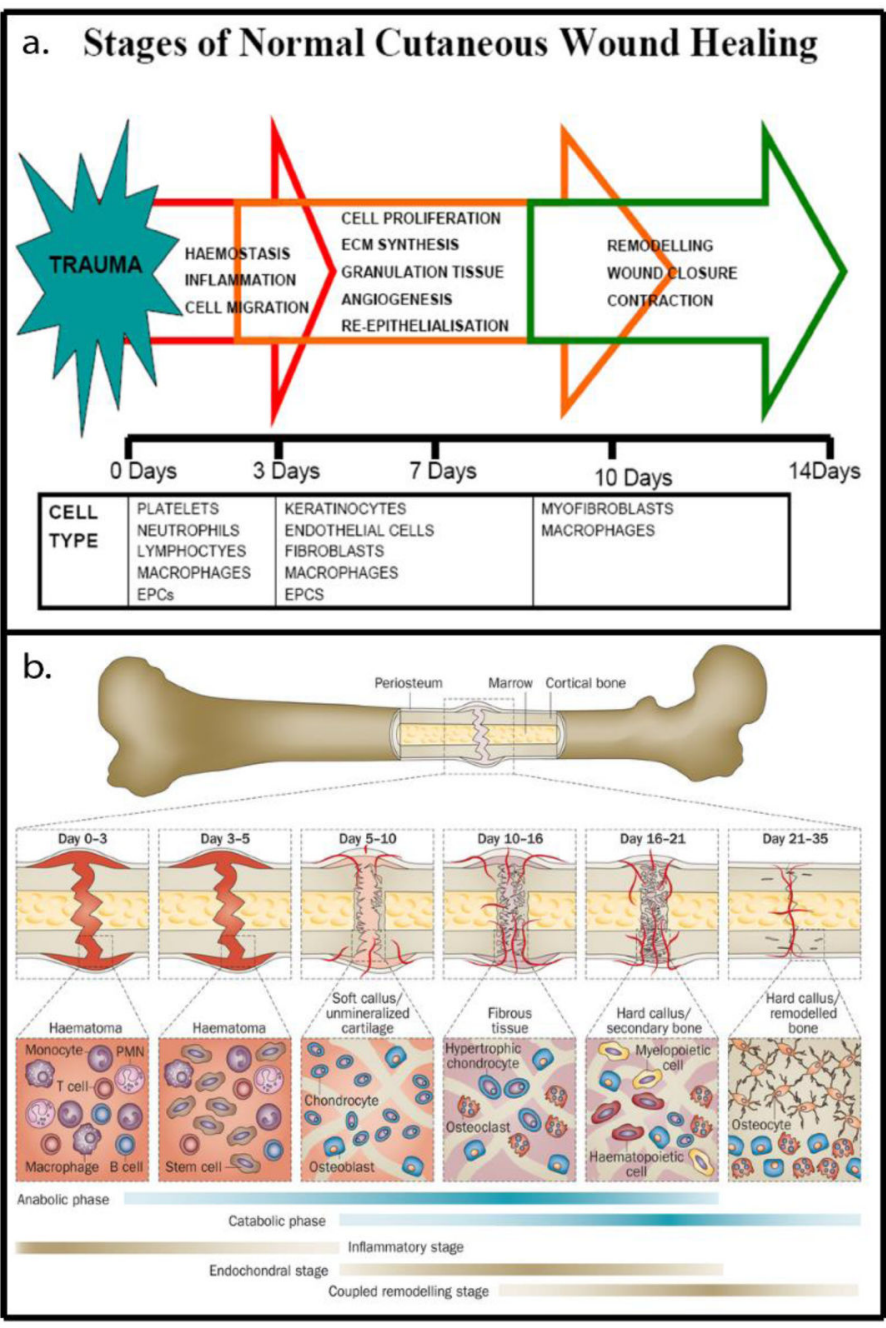

Fig. 1.

Timelines outlining successive stages in the processes of wound healing (a) and bone remodeling (b). Reprinted with permissions from (b) Ref. [4] and (a) Ref. [5]. (C 2011 O'Loughlin A, O'Brien T. Published in [5] under CC BY-NC-SA 3.0 license. Available from: http://dx.doi.org/10.5772/19070. 

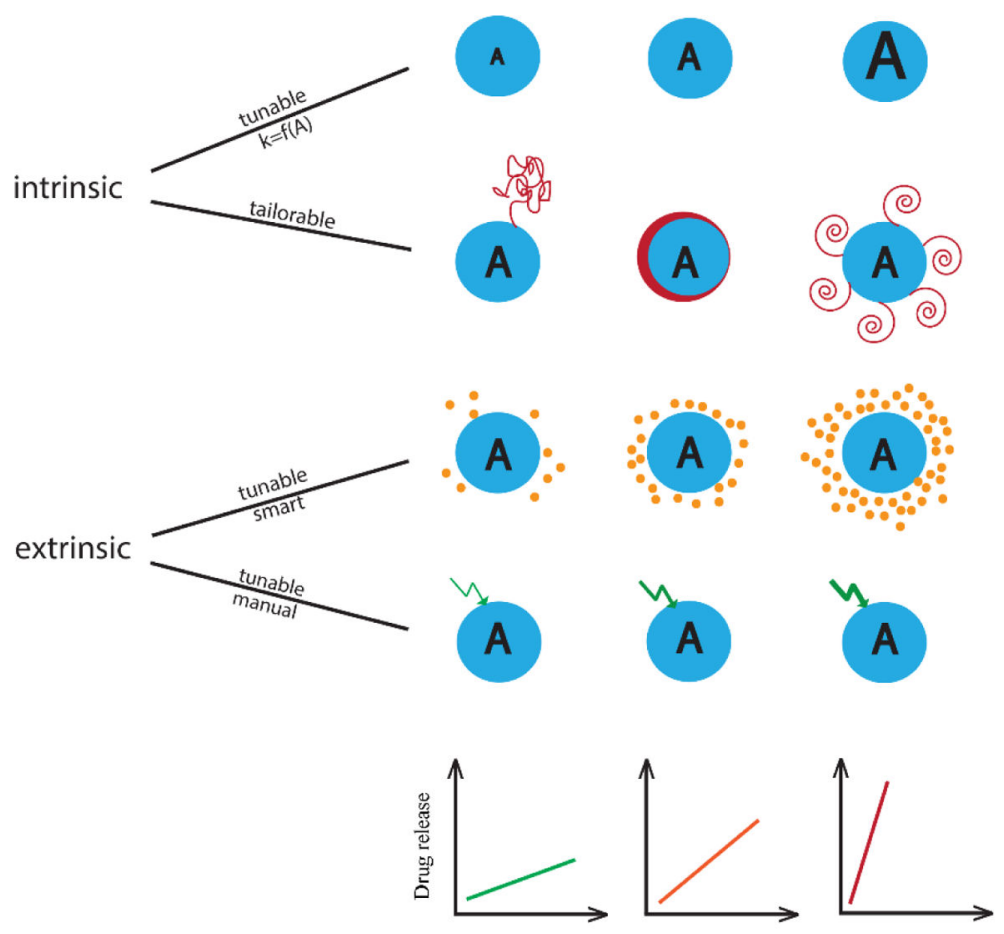

Fig. 2.

Four types of kinetically controlled drug release. Two intrinsic types of control (a, b) can be either tailorable (a) if the rate of release $(\mathrm{k})$ is directly proportional to a particular microstructural or compositional parameter of the carrier (A), or tunable (b) if the release is controlled by adding or subtracting different components of the carrier. Two extrinsic types of tunable release can be either smart, environmental (c) if the rate of release directly corresponds to the concentration of a chemical or biochemical marker in the environment surrounding the carrier or (d) manual if the rate of release is proportional to the intensity of an electromagnetic or acoustic signal produced by an externally operated device. A in the image refers to the microstructure or composition of the carrier, not the amount or concentration of the loaded drug. The three curves at the bottom refer to three kinetically distinct drug release profiles corresponding to the three different states of $\mathrm{A}$.

Expert Opin Drug Deliv. Author manuscript; available in PMC 2016 December 16. 

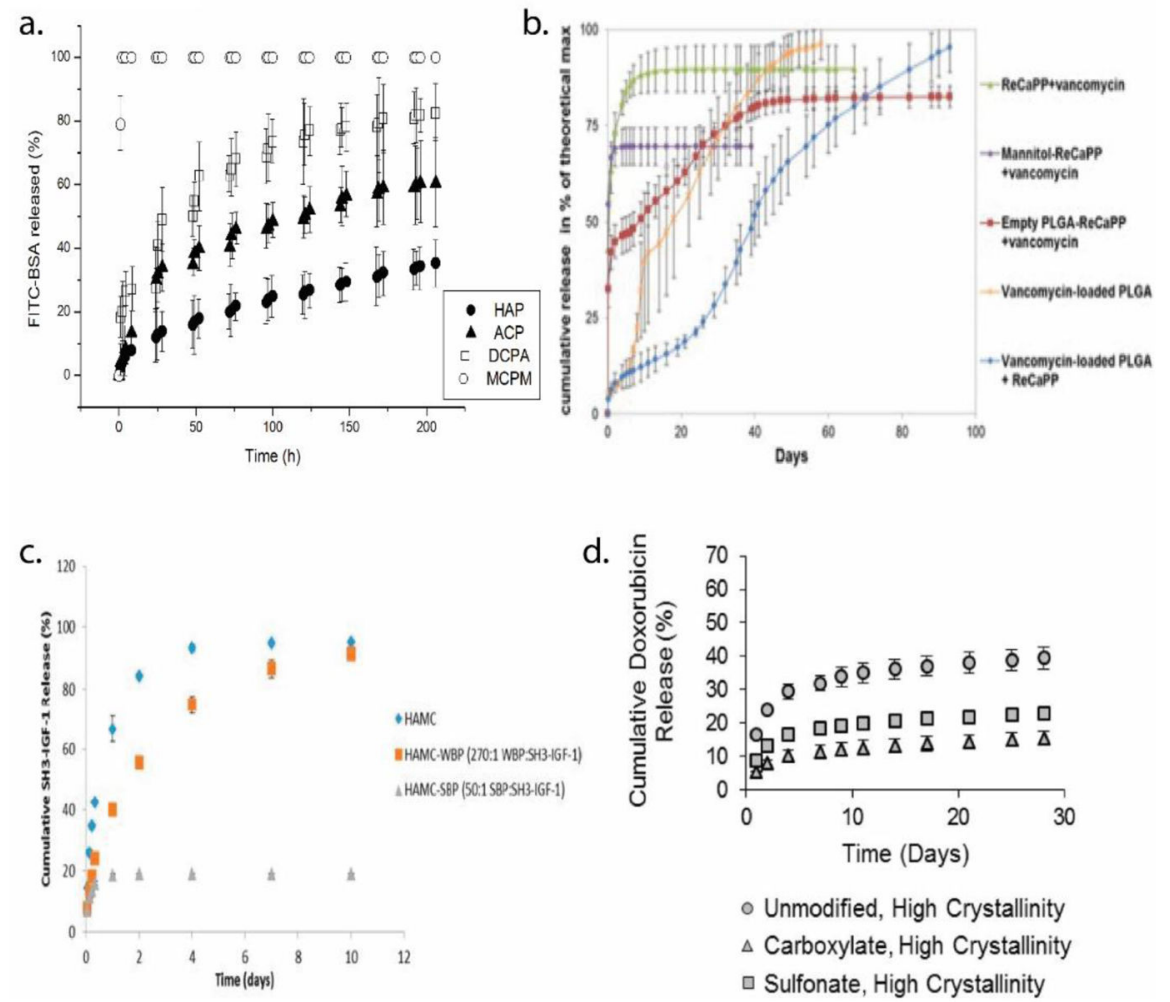

Fig. 3.

Different release profiles of: (a) bovine serum albumin depending on the qualitative nature of the mesoporous calcium phosphate carrier; (b) vancomycin depending on the qualitative, not quantitative change in the cement composition; (c) SH3-IGF-1 from HAMC depending on whether the hydrogel is bound to a weak binding peptide (WBP) or to a strong binding peptide (SBP); (d) doxorubicin depending on the qualitative modification of silk films as carriers. Reprinted with permissions from Refs. [21] (a), [25](b), [26](c), and [27] (d).

Expert Opin Drug Deliv. Author manuscript; available in PMC 2016 December 16. 
a.

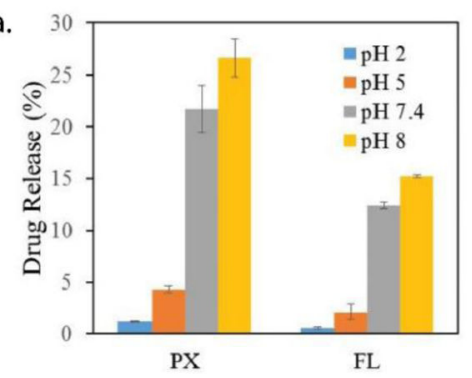

C.

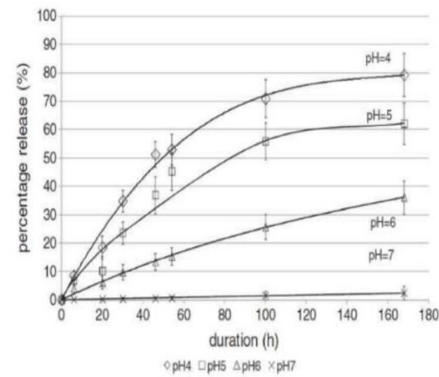

b.

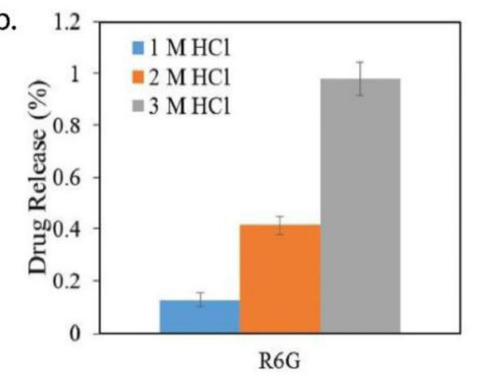

d.

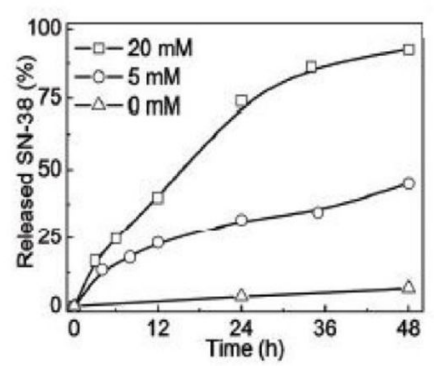

Fig. 4.

pH-triggered release of PX, FL (a) and R6G (b) from PPy nanoparticles. (c) pH-responsive release of gentamicin from $\mathrm{PEO}$-based polymeric nanoparticles covalently grafted onto titanium surface and functionalized with the antibiotic. (d) redox-responsive release of the antineoplastic drug, SN-38 from aniline-based polymeric nanoparticles. Reprinted with permission from Refs. [29] [30] and [31]. 
a.

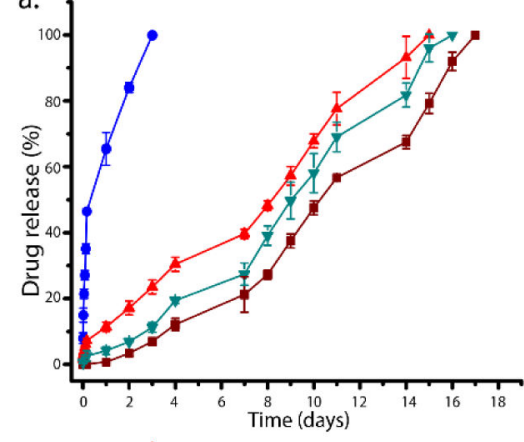

$\longrightarrow$ HAP1
$\rightarrow$ HAP2 HAP1/HAP2 50/50

C.

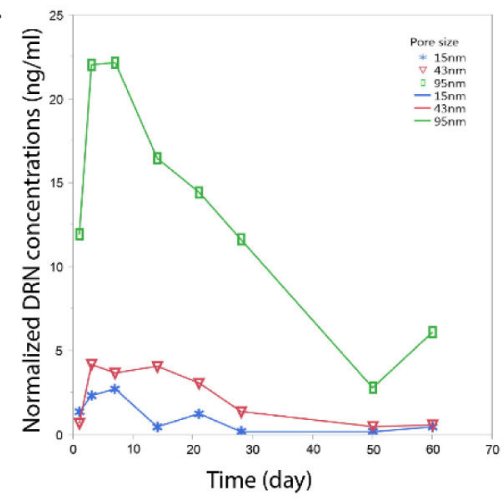

b.

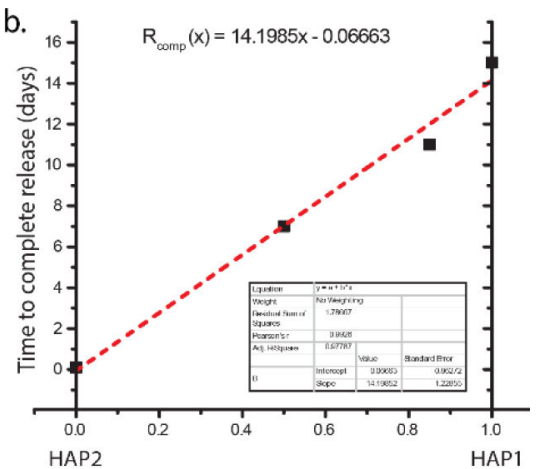

d.

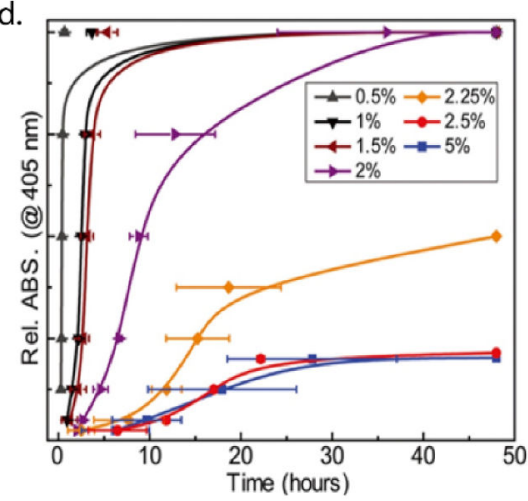

Fig. 5.

(a) Release of vancomycin from calcium phosphate pastes being tunable depending on the weight ratio between two of its components, HAP1 and HAP2. The linear function of the release rate versus the given weight ratio is shown in (b). (c) Release of daunorubicin from a silica-based drug delivery carrier with different nanopore sizes. (d) Release of horseradish peroxidase from gold-nanorod/PLGA microcapsules depending on the amount of PLGA in the precursor solution and the resulting microcapsule thickness. Reprinted with permission from Refs. [22] (a-b), [40] (c), and [57] (d). 


\section{Table 1}

Types of drugs delivered with the controlled release and the associated pharmaceutical product names.

\begin{tabular}{|c|c|c|}
\hline Delivered type of molecule & Pharmaceutical product & Reference \\
\hline Anti-inflammatory drugs & Surodex & [6] \\
\hline Corticosteroids & Ozurdex $®$, Retisert ${ }^{\circledR}$, Iluvien $®$ & [7] \\
\hline Anticancer chemotherapeutics & Gliadel, ThermoDox, Decapeptyl®, Trelstar ${ }^{\mathrm{TM}}$ & [8], [9], \\
\hline Contraceptives & Norplant, Jadelle & [10] \\
\hline Analgesics & DepoDur®, EXPAREL ${ }^{\circledR}$ & [11] \\
\hline Antipsychotics & Risperdal Consta ${ }^{\mathrm{TM}}$ & [12] \\
\hline Opioids & MS-Contin, Duragesic $®$ (Fentanyl), Butrans ${ }^{\circledR}$ & [13] \\
\hline Opioid antagonists & Vivitrol $^{\mathrm{TM}}$ & [14] \\
\hline Antivirals & Vitrasert & {$[15]$} \\
\hline Antibiotics & 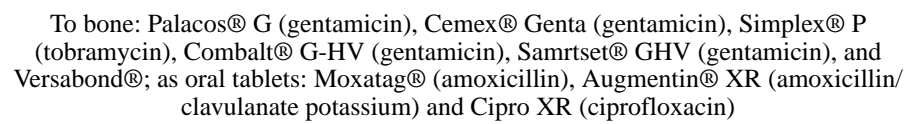 & [16], [17], [18] \\
\hline
\end{tabular}




\section{Table 2}

Examples of intrinsically tunable release carriers with intellectual property rights.

\begin{tabular}{|c|c|l|l|}
\hline Material & Filing date & Control parameter & Reference \\
\hline Organosiloxane-Poly(ethylene glycol) (PEG) & 1988 & $\begin{array}{l}\text { Amount of the water-in-oil emulsion mixed in } \\
\text { with the elastomer-forming composition }\end{array}$ & {$[66]$} \\
\hline Alpha-cyclodextrin and PEG-poly(propylene glycol)-PEG & 2001 & Copolymer weight ratio & {$[67]$} \\
\hline Polyurethane coating & 2002 & $\begin{array}{l}\text { Duration of the low-energy plasma } \\
\text { polymerization process }\end{array}$ & {$[68]$} \\
\hline $\begin{array}{c}\text { Poly(D, L-lactide-co-glycolide) (PLGA)-Hyaluronic acid } \\
\text { (HA) }\end{array}$ & 2007 & $\begin{array}{l}\text { Ratio of the hydrophilic (HA) to the } \\
\text { hydrophobic matrix (PLGA) }\end{array}$ & {$[69]$} \\
\hline PLGA coating & 2008 & $\begin{array}{l}\text { Weight percent of acetone in acetone/methyl } \\
\text { ethyl ketone spraying solution containing 2 wt. } \\
\text { \% polymer }\end{array}$ & {$[70]$} \\
\hline Polycaprolactone (PCL)-PLGA core-sheath fibers & 2009 & $\begin{array}{l}\text { Ratio between the feed rate of the inner (PCL) } \\
\text { and outer (PLGA) portion solutions during } \\
\text { electrospinning }\end{array}$ & {$[71]$} \\
\hline PCL coating & 2009 & PCL molecular weight in the 10 - 80 kDa range & {$[72]$} \\
\hline Hyaluronan and Methylcellulose (HAMC) & 2010 & MC content in HAMC, drug particle size & {$[73]$} \\
\hline Polystyrene-polyisobutylene & 2012 & $\begin{array}{l}\text { Conjugation degree of the prodrug to polymeric } \\
\text { carrier moieties }\end{array}$ & {$[74]$} \\
\hline
\end{tabular}




\section{Table 3}

Summary of the drug delivery carriers mentioned in the text and their categorization based on two major groups (intrinsic/extrinsic) and two subgroups (tunable/tailorable and smart/manual) described in Fig. 2, along with the key parameters for controlling the release kinetics.

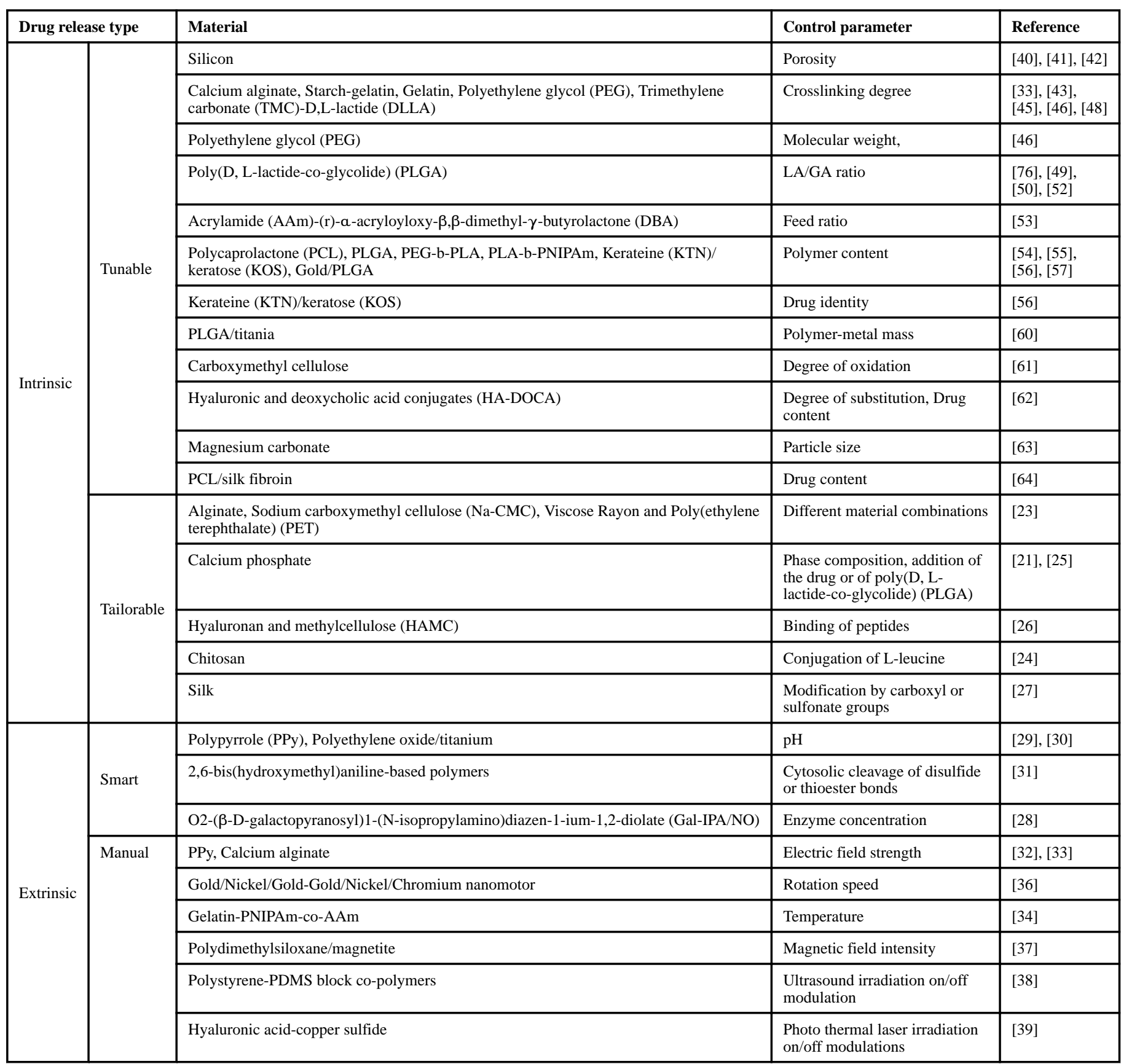

Expert Opin Drug Deliv. Author manuscript; available in PMC 2016 December 16. 